\title{
On the Communication Complexity of $t$-Intersection Problems in Generalized Boolean Algebras
}

\author{
Ulrich Faigle, WAlter Kern AND Boris SPIEker \\ Department of Applied Mathematics, University of Twente, P.O. Box 217, 7500 AE Enschede, \\ The Netherlands \\ e-mail: faigle@math.utwente.nl
}

\begin{abstract}
We consider the following game: Two players independently choose a chain in a partially ordered set. How many bits of information have to be communicated until at least one of the players knows whether the chains have exactly $t$ elements in common? This model generalizes the $t$ intersection problem for subsets of a finite set. We establish the deterministic communication complexity in general. For the special cases of generalized Boolean algebras, we present improved nondeterministic and probabilistic protocols that are of optimal order of complexity for classes with fixed width $q$.
\end{abstract}

Key Words: Communication complexity, generalized Boolean algebra, $t$-intersecting.

\section{Introduction}

The notion of communication complexity was introduced by Yao [1979] in order to derive lower bounds on the complexity of VLSI computations. For a thorough overview with applications to algorithmic combinatorics we refer to the survey of Lovász [1990] (see also Orlitsky and Gamal [1988] and Faigle and Turán [1990]). Without going into too many details, we briefly sketch the model.

Two players (I, II) have complete knowledge over a fixed $(0,1)$ matrix $M=$ $\left(m_{i j}\right)$, called the communication matrix. Player I selects a row $i$; and player II a column $j$. The players communicate bitwise in order to calculate the valuc of the matrix element $m_{i j}$. Each player has unbounded computing time. Communication ends as soon as one of the players has enough information to compute $m_{i j}$. How much information (measured in bits) needs to be exchanged in the worst case?

We first discuss the deterministic model:

The players agree in advance on a deterministic communication protocol which they will follow. The deterministic communication complex- 
ity $\operatorname{cc}(M)$ is the maximum number of bits needed, minimized over all deterministic protocols.

An important lower bound on $c c(M)$ is $\log _{2}(r(M))$, where $r(M)$ is the rank of $M$ over an arbitrary field (Mehlhorn and Schmidt [1982]). It was, in fact, conjectured that $\operatorname{cc}(M)=O\left(\log _{2} r(M)\right)$ holds as well. Recently, however, a counterexample to that conjecture has been found (cf. Raz and Spieker [1993]).

We now turn to the nondeterministic model:

Each player bases his transmission on guesses. A protocol is nondeterministic if the computation yields 0 for all choices $i, j$ with $m_{i j}=0$, and if $m_{i j}=1$, then there exists at least one guess sequence that would result in the correct answer. The nondeterministic communication complexity $\operatorname{cc}^{*}(M)$ is the maximum number of bits needed, minimized over all nondeterministic protocols.

It turns out that $\operatorname{cc}^{*}(M)=\left\lceil\log _{2} \operatorname{rect}(M)\right\rceil$, where $\operatorname{rect}(M)$ is the minimal number of "rectangles" (i.e.. submatrices) with only 1's needed to cover the 1's of the matrix $M$ (cf Lovász [1990]). We will use this fact in our approach for the estimation of $\mathrm{cc}^{*}(M)$ in the sequel.

Our paper deals with the communication complexity of the $t$-intersection problem in particular for generalized Boolean algebras $\mathscr{B}(n, q)$. These can be introduced as follows. First, for any $q, n \in \mathbb{N}$, let

$$
\mathscr{B}(n, q):=\{0,1, \ldots, q\}^{n}
$$

For $x, y \in \mathscr{B}(n, q)$ define

$$
\dot{x} \leq y \Leftrightarrow \forall i=1, \ldots, n:\left(x_{i} \neq 0 \Rightarrow x_{i}=y_{i}\right)
$$

and define the intersection $x \wedge y \in \mathscr{B}(n, q)$ by

$$
(x \wedge y)_{i}=\left\{\begin{array}{ll}
x_{i} & \text { if } x_{i}=y_{i}, \\
0 & \text { else }
\end{array} \quad \forall i=1, \ldots, n\right.
$$

Then $\mathscr{B}(n, q)$ becomes a so called semi-lattice. For $x \in \mathscr{B}(n, q)$ set

$$
|x|=\left|\left\{i: x_{i} \neq 0\right\}\right|
$$

The $t$-intersection problem $(t=0, \ldots, n)$ on $\mathscr{B}(n, q)$ can now be described as follows: players I and II get some elements $x, y \in \mathscr{B}(n, q)$, resp., and they are to 
find out whether $|x \wedge y|=t$. The corresponding communication matrix $M_{\mathscr{B}}^{t}$ has rows and columns indexed by elements $x, y \in \mathscr{B}(n, q)$ and in position $(x, y)$, the entry is 1 if and only if $|x \wedge y|=t$.

In Section 2 we develop a general lattice theoretic framework for computing the rank of certain classes of communication matrices. Basically, this is an extension to semi-lattices of Lovász's [1990] approach. As a conseqence, we are able to compute the rank of the matrix $M_{\mathscr{B}}^{t}$. Combined with the earlier mentioned log-rank lower bound, this implies that the trivial protocol, i.e. the protocol in which, say, player II communicates the index of his row, is optimal. This extends a previous result of Tamm [1991] for the case $q=1$.

Section 3 analyzes the nondeterministic communication complexity of the above problem. It turns out that for fixed $q \geq 1$ the nondeterministic complexity is of the same order of magnitude as the deterministic one, i.e., of order $n \log (q+1)$ if $t$ is not extremely big. For variable $q$ this remains true only for large values of $t$, say $t \geq$ const $\cdot n$. We exhibit an example for $t=0$ where the nondeterministic complexity cc* is of order $O(n \log \log q)$.

In Section 4 we deal with probabilistic communication complexity. We first briefly sketch the model. We calculate the probabilistic communication complexity of our problem for fixed $q$. For variable $q$ we give a protocol which leads to an upper bound on the probabilistic complexity of $O(n \log \log q)$, analogous to the one of Section 3 for nondeterministic computations.

\section{Deterministic Communication Complexity}

Let $M=\left(m_{x y}\right)$ be a communication matrix and denote by $h(M)$ the number of distinct columns of $M$. Then we observe

$$
\operatorname{cc}(M) \leq\left\lceil\log _{2} h(M)\right\rceil
$$

as a feasible protocol is given by the trivial protocol: player II specifies for player I the type of column he has chosen with $\left\lceil\log _{2} h(M)\right\rceil$ bits. (We assume here, w.l.o.g., that the number of distinct columns does not exceed the number of distinct rows in $M$ ). An often useful lower bound has been established by Mehlhorn and Schmidt [19821 in terms of the $\operatorname{rank} r(M)$ of $M$ :

$$
\left\lceil\log _{2} r(M)\right\rceil \leq \operatorname{cc}(M)
$$

It follows that the rank lower-bound is exact if $r(M)=h(M)$ holds. A method for computing the rank of certain classes of communication matrices is due to 
Lovász [1990] and is based on Wilf's [1968] representation of certain functions on ordered sets by Möbius algebra. Ahlswede et al. [1993] point out that Lovász's approach extends to a more general lattice-theoretic framework. What we need here is the fact that it suffices to work with semi-lattices. Let us give a brief outline.

Let $L=L(\leq, \wedge)$ be a (finite) semi-lattice, i.e., a (partially) ordered set $L$ such that for every $x, y \in L$ a unique infimum $x \wedge y \in L$ exists. Associate with $L$ the zeta-matrix $Z=\left(\zeta_{x y}\right)$ via

$$
\zeta_{x y}= \begin{cases}1 & \text { if } x \leq y \\ 0 & \text { otherwise }\end{cases}
$$

So $Z$ is just the incidence matrix of the order relation. It is easy to see that $Z$ has full rank. The inverse $Z^{-1}=\left(\mu_{x y}\right)$ is called the Möbius matrix of $L$. The following properties are not difficult to verify:

$$
\begin{aligned}
\mu_{x y} & =0 \text { if } x \not \leq y, \\
\mu_{x x} & =1, \\
\sum_{x \leq z \leq y} \mu_{x z} & = \begin{cases}0 & \text { if } x \neq y, \\
1 & \text { if } x=y .\end{cases}
\end{aligned}
$$

(For a more detailed discussion of Möbius algebra see, e.g., Aigner [1979]).

Let $f: L \rightarrow\{0,1\}$ be an arbitrary binary function and define the communication matrix $M(f)=\left(m_{x y}\right)$ via

$$
m_{x y}:=f(x \wedge y) \quad \forall x, y \in L
$$

Define the diagonal matrix $D=\left(d_{x y}\right)$ by

$$
d_{x y}= \begin{cases}\sum_{u \leq x} \mu_{x y} f(u) & \text { if } x=y, \\ 0, & \text { if } x \neq y\end{cases}
$$

Then Wilf noted

Lemma 2.1: $M(f)=Z^{T} D Z$. 
Sketch of proof: Assume $Z^{T} D Z=\left(a_{x y}\right)$. Then

$$
\begin{aligned}
a_{x y} & =\sum_{z \in L} \zeta_{z x} d_{z z} \zeta_{z y} \\
& =\sum_{z \leq x, y} d_{z z} \\
& =\sum_{z \leq x \wedge y} d_{z z} .
\end{aligned}
$$

In general, using the properties of $\mu_{x y}$ above, we have for any $v \in L$,

$$
\begin{aligned}
\sum_{w \leq v} d_{w w} & =\sum_{w \leq v} \sum_{u \leq w} \mu_{u w} f(u) \\
& =\sum_{u \leq v}\left[f(u) \sum_{u \leq w \leq v} \mu_{u w}\right] \\
& =f(v) .
\end{aligned}
$$

Hence $a_{x \wedge y}=f(x \wedge y)=m_{x y}$.

A direct consequence is

Theorem 2.1: $r(M(f))=\left|\left\{x \in L: d_{x x} \neq 0\right\}\right|$.

We are concerned with special functions $f$. To describe the appropriate framework, let us assume that the semi-lattice $L=L(\leq, \wedge)$ is equipped with a grading $|\cdot|: L \rightarrow \mathbb{N}$ satisfying for all $x, y \in L$,

$$
|x| \neq|y| \quad \text { whenever } x<y \text { holds } .
$$

For fixed $t \in \mathbb{N}$, consider the level indicator function $f_{t}: L \rightarrow\{0,1\}$, where

$$
f_{t}(x)= \begin{cases}1 & \text { if }|x|=t \\ 0 & \text { if }|x| \neq t\end{cases}
$$

Then the communication matrix $M=M\left(f_{t}\right)$ corresponds to the $t$-intersection problem in which the two players want to decide whether the grading of the intersection of their chosen elements is exactly $t$. 
Theorem 2.2: Let the semi-lattice $L$ be graded and assume that the Möbius matrix of $L$ satisfies for all $x \leq y$ in $L$,

$$
\mu_{x y} \neq 0 \quad \text { and the sign of } \mu_{x y} \text { depends only on the pair }(|x|,|y|)
$$

Then

$$
r\left(M\left(f_{t}\right)\right)=|\{x \in L:|x| \geq t\}|
$$

In particular, the trivial protocol is optimal for $M\left(f_{t}\right)$.

Proof: In view of Theorem 2.1, it suffices to show:

$$
\sum_{u \leq x} \mu_{u x} f_{t}(u) \neq 0 \Leftrightarrow|x| \geq t
$$

But this is an immediate consequence of the hypothesis with respect to the Möbius matrix of $L$ :

$$
\text { if }|u| \neq t \text { for all } u \leq x, \text { then } \sum_{u \leq x} \mu_{u x} f_{t}(u)=0
$$

if there is some $v \leq x$ with $|v|=t$, then sign $\sum_{u \leq x} \mu_{u x} f_{t}(u)=\operatorname{sign} \mu_{v x}$.

Thus the rank $r\left(M\left(f_{t}\right)\right)$ equals the number of non-trivial columns of $M\left(f_{t}\right)$. The Mehlhorn-Schmidt lower bound, therefore, implies that the trivial protocol is optimal.

In our context, examples satisfying the hypothesis of Theorem 2.2 can be obtained as follows. Given a finite (partially) ordered set $P$, we let $\mathscr{L}=\mathscr{L}(P)$ consist of all chains of $P$ with the set-theoretic containment as partial ordering. Then $\mathscr{L}$ is a semi-lattice with set-theoretic intersection as operation. It is easy to see that $\mathscr{L}=\mathscr{L}(\subseteq, \cap)$ is not a lattice unless $P$ consists of a single chain. Moreover, if $P$ is a chain, $\mathscr{L}(P)$ is isomorphic with the power set of $P$ and hence a Boolean algebra.

In general, if $X, Y \in \mathscr{L}(P)$ are chains of $P$ with $X \subseteq Y$, the interval

$$
[X, Y]=\{Z \in \mathscr{L}(P) \mid X \subseteq Z \subseteq Y\}
$$


is isomorphic with the Boolean algebra on $|Y|-|X|$ atoms, for which the Möbius matrix is well-known (cf. Aigner [1979]). So we conclude for all $X$, $Y \in \mathscr{L}(P)$,

$$
\mu_{x y}= \begin{cases}(-1)^{|Y|-|X|} & \text { if } X \subseteq Y, \\ 0 & \text { otherwise }\end{cases}
$$

which implies that the hypothesis of Theorem 2.2 holds for $\mathscr{L}(P)$ in general.

Generalized Boolean algebras $\mathscr{B}(n, q)$ (see the Introduction) can be interpreted in the present context in a straightforward manner:

Choose $n$ pairwise disjoint sets $A_{1}, A_{2}, \ldots, A_{n}$, all of the same cardinality $q$, and define an order relation on $P=A_{1} \cup \cdots \cup A_{n}$ via

$x<y \quad$ if there are $i<j$ with $x \in A_{i}$ and $y \in A_{j}$.

Then $\mathscr{L}(P)$ apparently is isomorphic with $\mathscr{B}(n, q)$. With the cardinality grading on $\mathscr{L}(P)$, Theorem 2.2 immediately yields for any $0 \leq t \leq n$ :

Corollary 2.1: Let $M=M(q, n, t)$ be the communication matrix associated with the t-intersection problem on the generalized Boolean algebra $\mathscr{B}(n, q)$. Then

$$
\operatorname{cc}(M)=\left\lceil\log _{2}|\{x \in \mathscr{B}(n, q):|x| \geq t\}|\right\rceil=\left\lceil\log _{2} \sum_{k=t}^{n}\left(\begin{array}{l}
n \\
k
\end{array}\right) q^{k}\right\rceil .
$$

We will address the nondeterministic communication complexity of the $t$ intersection problem on $\mathscr{B}(n, q)$ in the next section.

\section{Nondeterministic Communication Complexity}

In this section we study the nondeterministic communication complexity of the $t$-intersection problem on generalized Boolean algebras $\mathscr{B}(n, q)$. We first consider the problem for $q=1$, i.e., ordinary Boolean algebras $\mathscr{B}=\mathscr{B}(n):=\mathscr{B}(n, 1)$.

Theorem 3.1: The nondeterministic communication complexity, $\operatorname{cc}^{*}(n, t)$, of the $t$-intersection problem on $\mathscr{B}(n)$ equals the deterministic communication complexity 
up to a multiplicative constant, i.e., there is a constant $K>0$ such that

$$
\operatorname{cc}^{*}(n, t) \geq K \cdot \operatorname{cc}(n, t) \quad \text { for all } n \text { and } t
$$

The proof of Theorem 3.1, which will be given below, uses a result from Ahlswede et al. [1989]:

Lemma 3.1: Let $\mathscr{X}, \mathscr{Y} \subseteq \mathscr{B}(n)$ such that

$$
|X \cap Y|=t=\mathrm{const}
$$

for all $X \in \mathscr{X}, Y \in \mathscr{Y}$. Then $|\mathscr{X}| \cdot|\mathscr{Y}| \leq 2^{n}$.

Proof: (cf. Ahlswede et al. [1989]): Consider $\mathscr{B}(n)$ as a vector space over $G F(2)$ with inner product

$$
X \cdot Y=\sum_{i=1}^{n} X_{i} \cdot Y_{i} \quad \bmod 2
$$

Obviously, if $\mathscr{X}, \mathscr{Y} \subseteq \mathscr{B}(n)$ satisfy condition $(*)$, then

$$
X \cdot Y=t \bmod 2=\mathrm{const} \quad \forall X \in \mathscr{X}, Y \in \mathscr{Y} .
$$

Furthermore, if we take $X_{0} \in \mathscr{X}$, then

$$
\left(X_{0}+X\right) \cdot Y=0 \bmod 2 \quad \forall X \in \mathscr{T}, Y \in \mathscr{Y},
$$

i.e., $X_{0}+\mathscr{X}$ and $\mathscr{Y}$ are orthogonal. Hence

$$
\operatorname{dim}\left(X_{0}+\mathscr{X}\right)+\operatorname{dim}(\mathscr{Y}) \leq n
$$

which yields $|\mathscr{X}| \cdot|\mathscr{Y}| \leq 2^{n}$.

Now we are ready to present the

Proof of Theorem 3.1: We consider three cases: 
Case 1: $0 \leq t \leq n / 4$.

Let $M=M(n)=\left(m_{X Y}\right) \in \mathbb{R}^{\mathscr{B} \times \mathscr{B}}$ denote the matrix defined by

$$
m_{X Y}=|X \cap Y| \quad \forall X, Y \in \mathscr{B}=\mathscr{B}(n)
$$

(Note that $M(n)$, as defined here, is not a "communication matrix" in the strict sense because its entries are not restricted to $0,1 . M(n)$ represents the communication problem in a straightforward manner).

As mentioned in the Introduction, the nondeterministic communication complexity of the $t$-intersection problem equals $\left\lceil\log N_{t}\right\rceil$, where $N_{t}$ is the minimum number of $t$-rectangles, i.e., submatrices $M_{\mathscr{x} x y}$ of $M$ containing only $t$-entries, needed to cover all $t$-entries in $M$. Clearly, if we define the size of such a rectangle to be $|\mathscr{X}| \cdot|\mathscr{Y}|$, we get

$$
N_{t} \geq \frac{\text { number of } t \text {-entries in } M}{\text { maximum size of a } t \text {-rectangle }} .
$$

From Lemma 3.1 we see that the maximum size of a $t$-rectangle is bounded from above by $2^{n}$. On the other hand, it is straightforward to show that the total number of $t$-entries in $M$ equals $\left(\begin{array}{c}n \\ t\end{array}\right) \cdot 3^{n-t}$ for all $t=0, \ldots, n$. In fact, if one orders the rows and columns lexicographically, then the matrices $M(n)$ can be obtained recursively as follows:

$$
\begin{aligned}
& M(0)=[0], \\
& M(n+1)=\left[\begin{array}{cc}
M(n) & M(n) \\
M(n) & M(n)+1
\end{array}\right],
\end{aligned}
$$

where 1 is the "all-1-matrix" (of appropriate dimension). Thus, if $e(t, n)$ denotes the number of $t$-entries in $M(n)$, we conclude

$$
e(t, n+1)=3 \cdot e(t, n)+e(t-1, n),
$$

which implies $e(t, n)=\left(\begin{array}{c}n \\ t\end{array}\right) \cdot 3^{n-t}$, as claimed. Summarizing, we get for $t \leq n / 4$ :

$$
N_{t} \geq \frac{e(n, t)}{2^{n}} \geq\left(\frac{3^{3 / 4}}{2}\right)^{n} \geq 1.139^{n} \geq 2^{n / 6}
$$


Thus

$$
\mathrm{cc}^{*}(n, t) \geq \frac{1}{6} \cdot n \geq \frac{1}{6} \cdot \operatorname{cc}(n, t)
$$

Case $2: n / 4 \leq t \leq \frac{3}{4} \cdot n$.

In this case, observe that the submatrix of $M(n)$, consisting of all rows and columns indexed by elements $X, Y \in \mathscr{B}(n)$ which have exactly $t$ elements, is a $t$-multiple of the $\left(\begin{array}{l}n \\ t\end{array}\right) \times\left(\begin{array}{c}n \\ t\end{array}\right)$ identity matrix. Thus, in order to cover all $t$-entries of $M(n)$, we need at least $\left(\begin{array}{c}n \\ t\end{array}\right)$ rectangles. From this we conclude

$$
\operatorname{cc}^{*}(n, t) \geq \log \left(\begin{array}{l}
n \\
t
\end{array}\right) \geq t \geq n / 4 \geq \operatorname{cc}(n, t) / 4
$$

in this case.

Case 3: $t \geq \frac{3}{4} n$.

All $t$-entries of $M(n)$ are contained in a $k \times k$ submatrix of $M(n)$, where $k=$ $\left(\begin{array}{l}n \\ t\end{array}\right)+\cdots+\left(\begin{array}{l}n \\ n\end{array}\right) \leq 3 \cdot\left(\begin{array}{l}n \\ t\end{array}\right)$. Hence the deterministic complexity satisfies

$$
\operatorname{cc}(n, t) \leq\left\lceil\log \left[3 \cdot\left(\begin{array}{l}
n \\
t
\end{array}\right)\right]\right\rceil
$$

(cf. Section 2). Arguing as in the previous case, we get

$$
\operatorname{cc}^{*}(n, t) \geq \log \left(\begin{array}{l}
n \\
t
\end{array}\right) \geq \operatorname{cc}(n, t)-2
$$

Theorem 3.1 can be generalized to the $t$-intersection problem on $\mathscr{B}(n, q)$ for any fixed $q$. We only sketch the proof in the following. Let $q \in \mathbb{N}$ be fixed and let $M(n)=\left(m_{x y}\right) \in \mathbb{R}^{\mathscr{R}(n, q) \times \mathscr{B}(n, q)}$ be defined by

$$
m_{x y}:=|x \wedge y| \quad \forall x, y \in \mathscr{B}(n, q)
$$

It is then straightforward to verify that the number of $t$-entries in $M(n)$ equals 


$$
e(t, n)=\left(\begin{array}{l}
n \\
t
\end{array}\right) \cdot\left(q^{2}+q+1\right)^{n-t} \cdot q^{t}
$$

The maximum size of a $t$-rectangle can be bounded by the following result, which is easily derived from Theorem 1 in Ahlswede et al. [1989]:

Lemma 3.2: Let $\mathscr{X}, \mathscr{Y} \subseteq \mathscr{B}(n, q)$ such that

$$
|x \wedge y|=\left|x^{\prime} \wedge y^{\prime}\right| \quad \forall x, x^{\prime} \in \mathscr{X}, y, y^{\prime} \in \mathscr{Y}
$$

Then $|\mathscr{X}| \cdot|\mathscr{Y}| \leq(1+\lfloor q / 2\rfloor)^{n} \cdot(1+q-\lfloor q / 2\rfloor)^{n}$. (The maximum being attained, e.g., for $\mathscr{X}=\left\{\left(x_{1}, \ldots, x_{n}\right): 0 \leq x_{i} \leq\lfloor q / 2\rfloor \forall i\right\}$ and $\mathscr{Y}=\left\{\left(y_{1}, \ldots, y_{n}\right): y_{i}=0\right.$ or $\left.y_{i}>\lfloor q / 2\rfloor \forall i\right\}$.)

This gives an upper bound of $\left(\frac{q^{2}}{4}+q+1\right)^{n}$ on the maximum size of a rectangle. We conclude that for $t \leq n / 4$,

$$
\operatorname{cc}^{*}(n, t) \geq \log \left(\frac{\left(\begin{array}{l}
n \\
t
\end{array}\right) \cdot\left(q^{2}+q+1\right)^{n-t} \cdot q^{t}}{\left(\frac{q^{2}}{4}+q+1\right)^{n}}\right) .
$$

For $q \geq 2$, we have $\left(\frac{q^{2}}{4}+q+1\right) \leq\left(q^{2}+q+1\right) / 2$. This yields

$$
\operatorname{cc}^{*}(n, t) \geq \log \left[2^{n}\left(\begin{array}{l}
n \\
t
\end{array}\right) \cdot\left(\frac{q}{q^{2}+q+1}\right)^{t}\right] \geq n .
$$

On the other hand, the trivial deterministic protocol yields

$$
\operatorname{cc}(n, t) \leq n \cdot\lceil\log (q+1)\rceil
$$

Hence $\operatorname{cc}^{*}(n, t) \geq$ const $\cdot \operatorname{cc}(n)$ for fixed $q \geq 2$ and $t \leq n / 4$.

The case $t \geq n / 4$ is even slightly simpler than for $q=1$. Suppose $q \geq 2$ and $t \geq n / 4$. Then the vectors $x \in \mathscr{B}(n, q)$ with exactly $t$ non-zero entries give rise to a submatrix of $M(n)$ which is the $t$-multiple of the unit matrix of dimension $\left(\begin{array}{l}n \\ t\end{array}\right) \cdot q^{t}$. So, we need at least

$$
\left(\begin{array}{l}
n \\
t
\end{array}\right) \cdot q^{t} \geq\left(\begin{array}{c}
n \\
n / 4
\end{array}\right) \cdot q^{n / 4}
$$


rectangles in order to cover all $t$-entries of $M(n)$ for $t \geq n / 4$. This yields:

$$
\operatorname{cc}^{*}(n, t) \geq \log \left(\begin{array}{c}
n \\
n / 4
\end{array}\right) \cdot q^{n / 4} \geq \frac{n}{4} \cdot \log q \geq \frac{1}{4} \cdot \operatorname{cc}(n, t)
$$

Our proof shows that the claim of Theorem 3.1 remains valid for $t \geq n / 4$ (or any other linear bound for $t$ ) even if $q$ is considered as a variable. The case $t \leq n / 4$ is less clear. From Section 2 we conclude that $\operatorname{cc}(n, t)$ is of order $n \log q$. The following example, however shows that the nondeterministic complexity may be much smaller.

Example 3.1: Let $p_{1}, \ldots, p_{k}$ denote the first $k$ prime numbers and let $q=\prod_{i=1}^{k} p_{i}$. Consider the following nondeterministic protocol for $t=0$. Assume player I has a string $x=\left(x_{1}, \ldots, x_{n}\right) \in \mathscr{B}(n, q)$, and player II has a string $y=\left(y_{1}, \ldots, y_{n}\right) \in$ $\mathscr{B}(n, q)$. Suppose furthermore that $|x \wedge y|=0$. Player I now guesses for each $i=1, \ldots, n$ a prime factor $p$ of $q$ such that $r_{i}=x_{i} \bmod p \neq y_{i} \bmod p$. He then sends the index $j$ of this prime factor $p=p_{j}$ and the number $r_{i}$. This takes $O\left(\log k+\log p_{j}\right) \leq O\left(\log k+\log p_{k}\right)$ bits. Hence the total protocol uses $n \cdot O\left(\log k+\log p_{k}\right)$ bits. By the prime number theorem, the number of primes $\leq m$ is approximately greater or equal to $\frac{m}{\log m} \geq \sqrt{m}$. So there are at least $k$ primes not exceeding $k^{2}$. In other words, if $p_{1}, \ldots, p_{k}$ are the first $k$ prime numbers, then $p_{k} \leq k^{2}$. Consequently, we have

$$
\operatorname{cc}^{*}(n, 0)=O(n \log k)=O(n \log \log q)
$$

This should be seen in contrast to the deterministic complexity, which - as we have seen in Section 2 -is of order $n \log q$.

We leave it as an open problem to determine the exact order of magnitude of the nondeterministic communication complexity for $o(t / n)=0$, say, $t=$ const.

\section{Probabilistic Communication Complexity}

Probabilistic protocols are much like deterministic ones. The only difference is that the players follow a deterministic protocol $P$ which they have chosen $a t$ random from a set of different deterministic protocols. The rest of the computation works according to the specifications made by $P$ and is purely deterministic. 
Every deterministic protocol $P$ is, in fact, composed of two protocols $P_{I}$ and $P_{I I}$, resp., one for each of the players. $P_{v}$ is the protocol according to which player $v=\mathrm{I}$, II decides what to transmit. Let $\mathscr{P}_{v, m}$ be the family of protocols of length $\leq m$ for player $v$. A randomized protocol is a pair $\pi=\left(\pi_{I}, \pi_{I I}\right)$ where $\pi_{v}$ are probability distributions on $\mathscr{P}_{v, m}$. We say, $\pi$ has cost $m$.

Let $(x, y)$ be an input. To execute protocol $\pi$, each player generates a random $P_{v}$ according to the distribution $\pi_{v}$. Then I and II are following $\left(P_{I}, P_{I I}\right)$. Consider any input $(x, y)$ and $P=\left(P_{I}, P_{I I}\right) \in \mathscr{P}_{I, m} \times \mathscr{P}_{I I, m}$. We say, $P=\left(P_{I}, P_{I I}\right)$ computes correctly (the matrix element) $m_{x y}$ if both I and II decide on the answer $m_{x y}$ when $P$ is used for $(x, y)$. Let

$$
\lambda\left(M ; P_{I}, P_{I I} ; x, y\right)= \begin{cases}0 & \text { if }\left(P_{I}, P_{I I}\right) \text { computes } m_{x y} \text { correctly }, \\ 1 & \text { otherwise }\end{cases}
$$

For any input $(x, y)$, let $\lambda_{\pi}(M ; x, y)$ denote the probability that $\pi$ does not compute $m_{x y}$ correctly, i.e.,

$$
\lambda_{\pi}(M ; x, y)=\sum_{\left(P_{I}, P_{I I}\right) \in \mathscr{\mathscr { P }}_{1, m} \times \mathscr{P}_{I I, m}} \pi_{I}\left(P_{I}\right) \pi_{I I}\left(P_{I I}\right) \lambda\left(M ; P_{I}, P_{I I} ; x, y\right)
$$

The error probability for $\pi$ is defined to be the maximum error probability for any input, i.e. $\lambda_{\pi}(M)=\max _{(x, y)} \lambda_{\pi}(M ; x, y)$.

For any $0<\varepsilon<1 / 2$, let $\operatorname{cc}_{\varepsilon}(M)$, the probabilistic communication complexity of $M$, be the minimum cost of any randomized protocol $\pi$ with $\lambda_{\pi}(M) \leq \varepsilon$.

Theorem 4.1: (Kalyanasundaram and Schnitger [1992]) Let $S$ be the matrix $S=\left(s_{x y}\right)$ with the entries

$$
s_{x y}= \begin{cases}1 & \text { if } x \cap y=\varnothing \\ 0 & \text { otherwise }\end{cases}
$$

for all $x, y \subseteq\{0, \ldots, n-1\}$. Then it holds

$$
\mathrm{cc}_{\varepsilon}(S)=\theta(n) .
$$

Theorem 4.2: (Yao [1979]) Let $E$ be the $r \times r$ identity matrix, i.e., $E=(e q u(x y))$, where

$$
e q u(x y)=\left\{\begin{array}{ll}
1 & \text { if } x=y, \\
0 & \text { otherwise },
\end{array} \quad x, y \in\{1, \ldots, r\}\right.
$$


Then it holds:

$$
\mathrm{cc}_{\varepsilon}(E)=O(\log \log r)
$$

where $\varepsilon=\log \log r / \log r$. Furthermore, the protocol used in the proof never errs if the players decide on " $x \neq y$ ".

Let $q$ be fixed and assume there is a probabilistic protocol $P$ which computes

$$
f_{t}(x \wedge y)= \begin{cases}1 & \text { if }|x \wedge y|=t \\ 0 & \text { otherwise }\end{cases}
$$

for $x, y \in \mathscr{B}(n, q)$ with a small error probability, $<1 / 4$, say, in time $g(n, q)$.

We claim that $g(n, q)=\Omega(n)$.

Indeed, if $g(n, q)$ were smaller than $n$ by more than a constant multiple, there would exist a probabilistic protocol $P$ for the matrix $S$ of Theorem 4.1 with a performance of $O(g(n, q))$ bits. Let $A=\left(x_{1}, \ldots, x_{n}\right), B=\left(y_{1}, \ldots, y_{n}\right) \in\{0,1\}^{n}$. Transform $A$ and $B$ resp. in $\widetilde{A}=\left(x_{1}, \ldots, x_{n}, x_{n+1}, \ldots, x_{n+t}\right)$ and $\widetilde{B}=\left(y_{1}, \ldots, y_{n}\right.$, $\left.y_{n+1}, \ldots, y_{n+t}\right)$ with $x_{n+1}=y_{n+1}=\cdots=x_{n+t}=y_{n+t}=1$. Follow now the protocol $P$ with inputs $\tilde{A}$ and $\tilde{B}$ resp. Then

$$
A \cap B=\varnothing \Leftrightarrow|\tilde{A} \cap \tilde{B}|=t
$$

For every $A, B \in\{0, \ldots, n-1\}$ the players can decide the set-intersection problem in time $O(g(n, q))(t \leq n)$, which contradicts Theorem 4.1.

As in the nondeterministic case, we have to distinguish between fixed and variable $q$. Let $M=\left(m_{x y}\right)$, where

$$
m_{x y}=f_{t}(x \wedge y) \quad \forall x, y \in \mathscr{B}(n, q)
$$

The following protocol for $q$ such that $\log \log q / \log q \leq 1 / 8$ and $t=0$ shows that

$$
\mathrm{Cc}_{\varepsilon}(M)=2 n \cdot O(\log \log q)
$$

in comparison to the $\theta(n \log q)$ bits which are needed in the deterministic case. First we state a standard probabilistic fact. 
Lemma 4.1: Let $\varepsilon \leq 1 / 8$ and consider $2 n$ Bernoulli trials each with probability of success of $1-\varepsilon$. Then

Prob (less than $n$ successes $) \leq \frac{1}{2^{n}}$.

Protocol: Let $(x, y)$ be an input. Let $L=\left\{i \in\{1, \ldots, n\}: x_{i} \neq 0\right\}$. Set $j=0$, $k_{0}=|L|$ and $r=0$.

1. Player I sends $n$ bits to player II in order to transmit $L$.

2. For $l=1, \ldots, k_{j}$ :

(i) $r:=r+1$;

(ii) if $r>2 n$, STOP;

(iii) following Yao's protocol, decide whether equ( $\left.x_{i_{i}}, y_{i_{l}}\right)=0$, and, if so, set $L:=L-\{l\}$

(iv) if $L=\varnothing$, STOP.

3. Set $j:=j+1, k_{j}=|L|$ and let $L=\left\{i_{1}, \ldots, i_{k_{j}}\right\}$. Goto 2 .

4. The players decide that $f_{0}(x \wedge y)=0$ if $k_{j}>0$, they decide on the answer 1 otherwise.

Because our protocol follows Yao's protocol in step 2 (iii), Theorem 4.2 implies that the number of bits transmitted is of order $n \log \log q$. If the players decide on $f_{0}(x \wedge y)=1$ there is no error. What is the error probability Prob(decide on $\left.f_{0}(x \wedge y)=0 \mid f_{0}(x \wedge y)=1\right)$ ?

Consider the (at most $2 n$ ) queries

$e q u\left(x_{l}, y_{l}\right)=?$

Let $f_{0}(x \wedge y)=1$. In one query, with probability of $1-\frac{\log \log q}{\log q}=1-\varepsilon$, we reveal the (correct) answer $0=e q u\left(x_{l}, y_{l}\right)$, i.e. $x_{l} \neq y_{l}$. So, if we make an error in our final decision, the following has happened: in $2 n$ Bernoulli trials with success probability $1-\varepsilon$, we have observed less than $n$ successes. By Lemma 4.1, however, the probability for this to occur is at most $\frac{1}{2^{n}}$.

Without going into details, let us finally mention that it is possible to extend the above protocol to the case $t \leq n / \log n$. The number of bits exchanged will still be bounded by $O(n \log \log q)$. In fact, our protocol will not only give an answer to the question whether $|x \wedge y|=t$, but will also exhibit the exact number of intersecting positions with high probability. 


\section{References}

Ahlswede R, Cai N, Zhang Z (1989) A general 4-words inequality with consequences for 2-Way communication complexity. Adv Appl Math 10:75-94

Ahlswede R, Cai N, Tamm U (1993) Communication complexity in lattices. In: Proceedings of the 3rd Twente Workshop on Graphs and Combinatorial Optimization, Memorandum 1132, Faculty of Applied Mathematics, University of Twente, Enschede, The Netherlands, 191-194

Aigner M (1979) Combinatorial theory. Springer Verlag

Faigle U, Turán G (1990) Communication complexity. Computing Suppl 7:141-154

Kalyanasundaram B, Schnitger G (1992) The probabilistic communication complexity of set intersection. SIAM J Disc Math 5 4:545-557

Lovász L (1990) Communication complexity: A survey . In: Korte B et al. (eds) Patbs, Flows and VLSI-Layout Springer Verlag, 235-266

Mehlhorn K, Schmidt EM (1982) Las Vegas is better than determinism in VLSI and distributed computing. Proc 14th ACM STOC, 330-337

Orlitsky A, El Gamal A (1988) Communication complexity. In: Abu-Mostafa YS (ed) Complexity in Information Theory Springer Verlag

Raz R, Spieker B (1993) On the 'log-rank' conjecture in communication complexity. Proc. FOCS 93, Palo Alto, 1993, 168-176

Tamm U (1991) Deterministic communication complexity of the set-intersection function. Preprint 91-077, SFB 343, Universität Bielefeld

Wilf HS (1968) Hadamard determinants, möbius functions and the chromatic number of a graph. Bull Amer Math Soc 74:960-964

Yao AC (1979) Some complexity questions related to distributive computing. Proc 11th ACM STOC, 209-219 